\title{
Fuzzy Multiobjective Optimal Power Flow Based on Modified Artificial Bee Colony Algorithm
}

\author{
Xuanhu He and Wei Wang \\ National Active Distribution Network Technology Research Center, Beijing Jiaotong University, No. 3 Shang Yuan Cun, \\ Haidian District, Beijing 100044, China
}

Correspondence should be addressed to Xuanhu He; 11117367@bjtu.edu.cn

Received 13 January 2014; Accepted 27 February 2014; Published 8 April 2014

Academic Editor: Changzhi Wu

Copyright (c) $2014 \mathrm{X}$. He and W. Wang. This is an open access article distributed under the Creative Commons Attribution License, which permits unrestricted use, distribution, and reproduction in any medium, provided the original work is properly cited.

This paper presents a modified artificial bee colony (MABC) algorithm to solve optimal power flow (OPF) problem. In the proposed $\mathrm{MABC}$ algorithm, the searching operation for new food source of artificial bee colony $(\mathrm{ABC})$ algorithm is replaced with mutation and crossover operation of differential evolution (DE) algorithm to improve exploitation capacity. The OPF objective functions involve minimization of total fuel cost of generating units, minimization of emission of atmospheric pollutants, minimization of active power losses, and minimization of voltage deviations. The fuzzy satisfaction-maximizing method is utilized to convert the multiobjectives problem into single objective problem. The proposed approach is applied to the OPF problem on IEEE 30-bus test system. And the results are compared with those obtained by other heuristic algorithms, which demonstrate that the MABC algorithm not only has a better exploration capacity but also possesses stronger exploitation capacity and can effectively solve the OPF problem.

\section{Introduction}

Optimal power flow (OPF) was first proposed by French scholar Carpentier in the 1960s. Its definition can be described as the optimal power flow distribution of power system with fixed structure parameters and loads, which optimizes the objective functions by optimal settings of the OPF control variables, while at the same time satisfying various constraints $[1,2]$. OPF problem has received much attention by the academics due to the fact that it is an effective analysis tool for the safe operation and economic dispatch of power system.

On the aspect of choosing OPF objective function, Paranjothi and Anburaja in [3] established the single objective OPF model which considered the minimization of total fuel cost as objective function. The results illustrated that the economics of power system could be further improved in some extent. Because the active power loss caused by transmission and distribution and the emission cost brought by the some generators affect the economics of the power system [4], the OPF objective functions which are established from the aspect of system economics also need to consider these two factors. In order to further improve the system stability, Pouyan et al. in [5] proposed the OPF objective function which furthermore considered voltage stability index, and the obtained optimization scheme considers both economics and stability of power system. The multiobjective OPF model can comprehensively reflect the operation performance of the optimized power system, which improves not only the economy of power system but also the stability of operation.

The weighting method [6] and fuzzy mathematics method [7] et al. are usually used to handle each objective weight of multiobjective optimization problem. The weighting method adopts weighting coefficients to treat each objective function to form a single objective. This method can solve multiobjective OPF effectively, but the weighting coefficients are determined either by subjective settings or by several tests for optimal scheme to get a more satisfactory function value. The fuzzy mathematics method uses membership function to make objective function fuzzification to form the fuzzy multiobjective OPF. There is no need to use weighting 
coefficients to solve multiobjective problem in fuzzy mathematics method, and the solution is obtained objectively.

The OPF problem is considered as complex multiconstraints, nonlinear, and noncontinuous optimization problem regardless of OPF model with single objective or multiobjective function. Many classical algorithms such as linear programming [8], quadratic programming [9], simplified gradient method [10], Newton method [11], and interior point method [12] have been widely applied to solve the OPF problem. These algorithms are taking an order or second order gradient of objective functions as the main information to search for the optimal solution and have relatively rapid calculation. They are suitable for online calculation but not suitable for optimization problem with discrete variables. The heuristic algorithms are novel algorithms for solving the optimization problem. And the typical heuristic methods include genetic algorithm (GA), particle swarm optimization (PSO), differential evolution (DE), artificial immune algorithm, and artificial bee colony (ABC) algorithm [13-17]. These algorithms are based on multipoint stochastic searching, and they can effectively solve optimization problem with discrete variables. Their global convergence capacity is better than classical algorithms.

The ABC algorithm possesses superior performance on many benchmark functions compared to other heuristic algorithms [18]. It was proposed by Karaboga in 2005 and it simulated the intelligent foraging behavior of honeybee swarms [19]. Its advantages include easy implementation and better global search ability. The ABC algorithm was applied to the power system network reconfiguration problem which considered the power loss as the optimization objective function for the first time in the literature [20] and was simulated on test system. The results show that the power loss of reconfiguration system by the $\mathrm{ABC}$ algorithm is less and running time is shorter than other algorithms. In [21], the authors utilized the ABC algorithm to solve the economic power dispatch problem, and the proposed method was applied to the test system. The results indicate that the exploration capacity of ABC algorithm was better than other heuristic algorithms.

The ABC algorithm is also easily to get trapped in local optima as a heuristic algorithm [22]. In order to overcome the shortage of $\mathrm{ABC}$ algorithm, the scholars have proposed some improved ABC algorithms [23-25]. Gao and Liu in [26] proposed the new solution search equation of $\mathrm{ABC}$ algorithm which was inspired by the DE/best/1 algorithm and adopted the chaotic systems and opposition-based learning method to generate the initial population. In [27] some modified search equations were proposed and the orthogonal learning strategy was applied to discover the useful information from the search experiences to improve the optimization performance of $\mathrm{ABC}$ algorithm. However, the exploration capacity and exploitation capacity are not very well balanced in some improved algorithms. For comprehensively improving the exploration and exploitation capacity, the paper proposes a modified $\mathrm{ABC}$ algorithm named MABC algorithm whose searching operation for new food source of $\mathrm{ABC}$ algorithm is replaced with mutation and crossover operation of $\mathrm{DE}$ algorithm and uses the MABC algorithm to solve OPF problem. For comprehensively considering the economics and stability of the power system, the OPF objective functions involve minimization of total fuel cost, minimization of emission, minimization of active power loss, and minimization of voltage deviation. The fuzzy satisfaction-maximizing method is utilized to convert the multiobjective problem into single objective problem to form fuzzy multiobjective OPF. Finally, the fuzzy multiobjective OPF based MABC algorithm is tested on the standard IEEE 30-bus test systems. The simulation results are compared with those obtained by other heuristic methods, and they demonstrate that MABC algorithm possesses not only better exploration capacity but also stronger exploitation capacity.

\section{OPF Mathematical Model}

The main purpose of the OPF is to determine the optimal control variables values for minimizing one or more objective functions while satisfying the several equality and inequality constraints. Its mathematical model [28] can be established as

$$
\begin{array}{ll}
\min & f(x, u) \\
\text { s.t. } & g(x, u)=0, \quad h(x, u) \leq 0,
\end{array}
$$

where $f$ is the objective function to be optimized, $g$ is the equality constraints representing nonlinear power flow equations, and $h$ is the system operating constraints; $x$ is the vector of dependent state variables including slack bus generated active power, generator reactive power output, load $(P Q)$ bus voltage, and transmission line flow; $u$ is the vector of independent control variables including generator active power output, generator bus voltage, transformer tap settings, and shunt VAR compensation.

The paper considers four optimization objectives of OPF. Their mathematical models and OPF constraints are described as follows.

\subsection{OPF Objective Function}

2.1.1. Minimization of Total Fuel Cost. The fuel cost of generating units is one of the important factors affecting the system economic. And how to allocate the active power output at each generating unit decides the total fuel cost under the conditions of fixed demand active power. Therefore, the total fuel cost is considered as one of the OPF objective functions, and it can be expressed as

$$
\mathrm{FC}=\sum_{i=1}^{N_{G}} a_{i} P_{G i}^{2}+b_{i} P_{G i}+c_{i},
$$

where FC is the total fuel cost; $a_{i}, b_{i}$, and $c_{i}$ are the fuel cost coefficients of the $i$ th generator; $P_{G i}$ is the active power output of thermal unit $i ; N_{G}$ is the number of generators.

2.1.2. Minimization of Total Emission. The total emission of atmospheric pollutants is caused by the operation of fossil-fueled thermal generation. And the treatment cost of 
pollutants will be produced, which have an influence on the economical operation of power system. Therefore, the total emission is also considered as one of objective functions, and it can be described as

$$
\mathrm{EM}=\sum_{i=1}^{N_{G}} \alpha_{i} P_{G i}^{2}+\beta_{i} P_{G i}+\gamma_{i},
$$

where EM is the total emission; $\alpha_{i}, \beta_{i}$, and $\gamma_{i}$ are the emission coefficients of the $i$ th generator.

2.1.3. Minimization of Total Power Loss. Active power loss is produced by the transmission and distribution in power system. It is an important measurable index of the economic operation. Smaller active power loss will generate the better economic benefits; therefore, the total power loss is considered as one of objective functions and it can be expressed as

$$
P_{\text {loss }}=\sum_{i, j=1}^{N} G_{i j}\left(V_{i}^{2}+V_{j}^{2}-2 V_{i} V_{j} \cos \theta_{i j}\right) \text {, }
$$

where $P_{\text {loss }}$ is active power loss; $V_{i}, V_{j}$ are the voltage magnitudes at bus $i$ and bus $j$, respectively; $G_{i j}, \theta_{i j}$ are the branch conductance and phase angles difference between bus $i$ and bus $j$, respectively; $N$ is the total number of nodes.

2.1.4. Minimization of Voltage Deviation. The stability of power system is considered as one of the optimization objectives besides the economics of system. The bus voltage deviation is an important index of voltage stability, and it can measure size of the deviations from 1.0 per unit and represent the overall level of voltage stability. This objective function can be described as

$$
\Delta V=\sum_{i=1}^{N_{P Q}}\left|V_{i}-1.0\right|
$$

where $N_{P Q}$ is the number of $P Q$ nodes and 1.0 is per unit.

2.2. OPF Constraints. It can be seen that the control variables and state variables must be satisfied with some constraints to make the power system on the stability operation from the definition of OPF. The OPF constraints of power system include equality constraints and inequality constraints.

The equality constraints are the power flow equations, and they can be described as

$$
\begin{gathered}
P_{G i}-P_{L i}=V_{i} \sum_{j=1}^{N} V_{j}\left(G_{i j} \cdot \cos \theta_{i j}+B_{i j} \cdot \sin \theta_{i j}\right) \\
Q_{G i}-Q_{L i}=V_{i} \sum_{j=1}^{N} V_{j}\left(G_{i j} \cdot \sin \theta_{i j}-B_{i j} \cdot \cos \theta_{i j}\right),
\end{gathered}
$$

where $P_{G i}, Q_{G i}$ are active power and reactive power outputs of generating units at bus $i$, respectively; $P_{L i}, Q_{L i}$ are the demanded active power and reactive power of loads at bus $i$, respectively; $G_{i j}, B_{i j}$, and $\theta_{i j}$ are transfer conductance, susceptance, and voltage angle difference between bus $i$ and bus $j$, respectively; $V_{i}, V_{j}$ are voltage magnitude at bus $i$ and bus $j$, respectively; $N$ is total number of nodes.

The inequality constraints include control variables constraints and state variables constraints in the OPF model. And the control variables constraints can be described as

$$
\begin{aligned}
P_{G i \min } & \leq P_{G i} \leq P_{G i \max }, \\
V_{G i \min } & \leq V_{G i} \leq V_{G i \max }, \\
T_{t k \min } & \leq T_{t k} \leq T_{t k \max }, \\
Q_{c j \text { min }} & \leq Q_{c j} \leq Q_{c j \text { max }},
\end{aligned}
$$

where $P_{G i}, V_{G i}, T_{t k}$, and $Q_{c j}$ are active power output of the generator, terminal voltage of the generator, transformer tap settings, and reactive power compensation capacity, respectively. They are automatically satisfied by setting the constraint boundary.

The state variables constraints can be described as

$$
\begin{gathered}
V_{D i \min } \leq V_{D i} \leq V_{D i \max }, \\
Q_{G j \min } \leq Q_{G j} \leq Q_{G j \max }, \\
S_{L k}<S_{L k \text { max }},
\end{gathered}
$$

where $V_{D i}, Q_{G j}$, and $S_{L k}$ are the load bus voltage magnitudes, reactive power output of generator, and transmission line flow, respectively. They are satisfied by penalty functions that are brought in the objective functions.

\section{Fuzzy Multiobjective OPF}

The multiobjective OPF model is established, which involves the minimization of total fuel cost, the minimization of total emission, the minimization of power loss, and the minimization of voltage deviation. For objectively considering the weight of each optimization objective, the linear membership function is used to make the objective functions fuzzification to form the fuzzy multiobjective function OPF. The linear membership function can be described as

$$
\begin{aligned}
& \mu_{i}(x) \\
& = \begin{cases}1 & f_{i}(x)<c_{i \min } \\
\frac{c_{i \max }-f_{i}(x)}{c_{i \max }-c_{i \min }} & c_{i \min }<f_{i}(x)<c_{i \max } \\
0 & f_{i}(x)>c_{i \max },\end{cases}
\end{aligned}
$$

where $f_{i}(x)$ is $i$ th objective function of fuzzy multiobjective OPF; $\mu_{i}(x)$ is the membership function of $f_{i}(x) ; m$ is the number of objective functions; $c_{i \max }, c_{i \min }$ are upper and lower limit values of $f_{i}(x)$, respectively, $c_{i \text { min }}$ is the optimal value obtained by single objective OPF for each objective function, and $c_{i \max }$ is initial value of each objective function. The curves of each membership function by (9) are shown in Figure 1.

Abscissa [4] represents each objective function value of fuzzy multiobjective OPF, and the ordinate represents the 


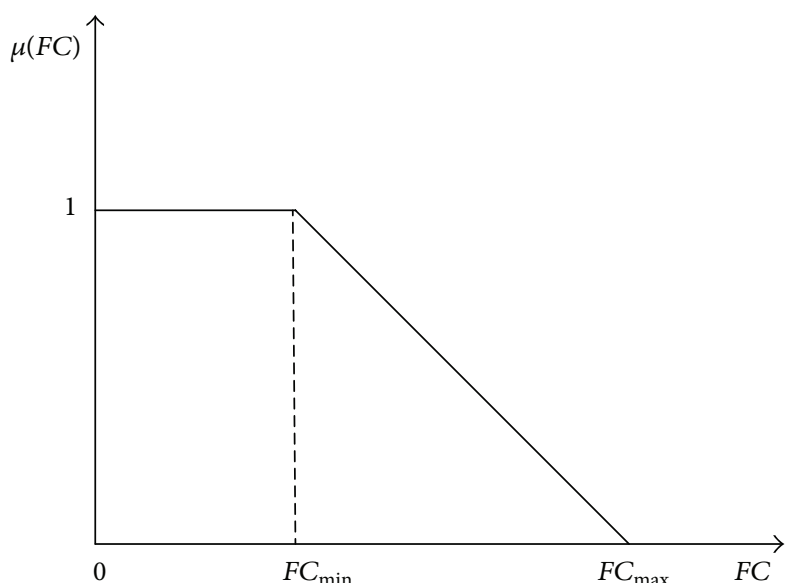

(a) Membership function of total fuel cost

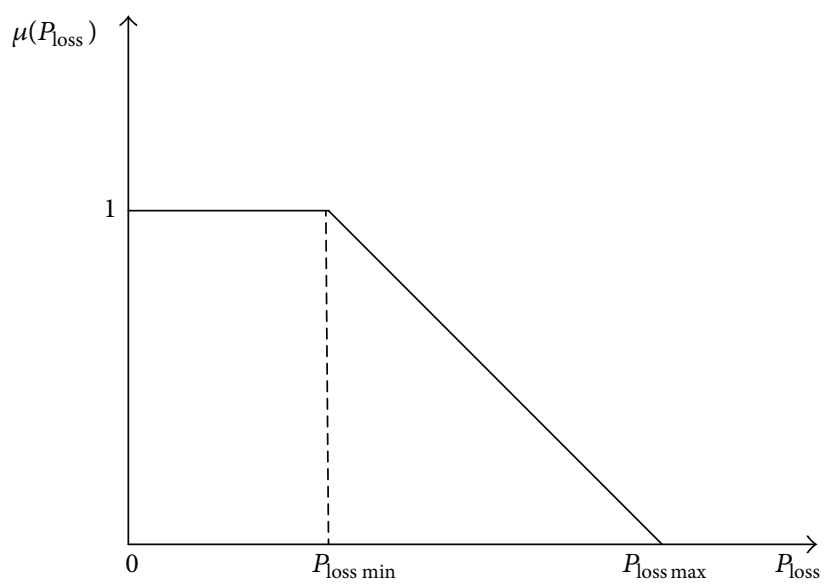

(c) Membership function of power loss

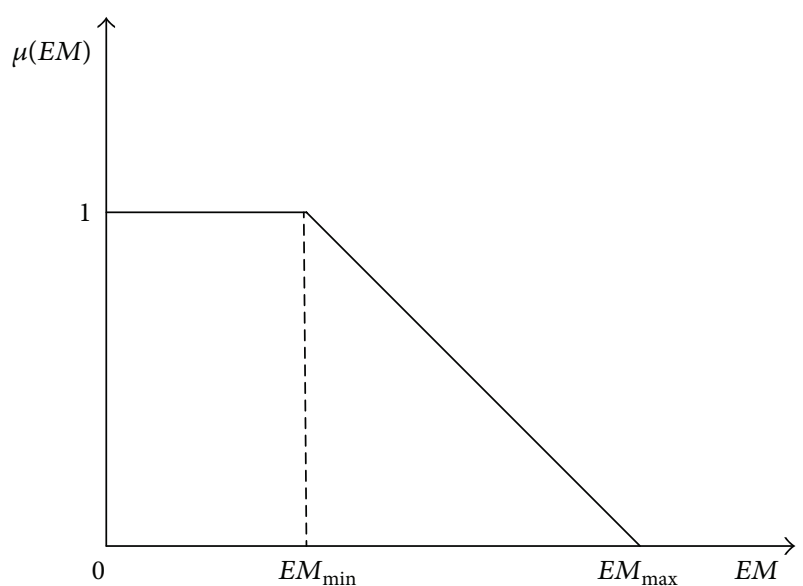

(b) Membership function of total emission

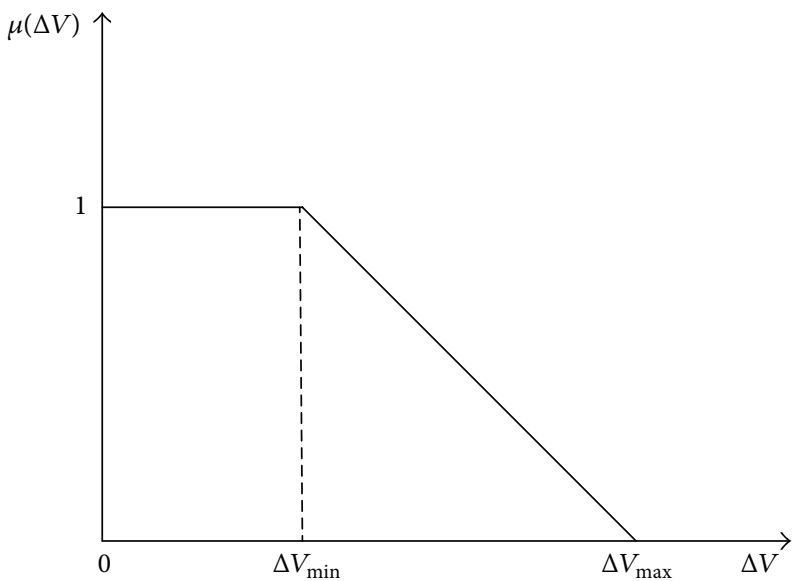

(d) Membership function of voltage deviation

FIGURE 1: Membership functions of each subobjective.

membership function value of each optimization objective at (a), (b), (c), and (d) in Figure 1. From (9) or Figure 1, it can be seen that the larger the membership function value, the smaller the corresponding objective function. The objective function $\mu_{D}(x)$ of fuzzy multiobjective OPF is chosen from membership function $\mu_{i}(x)$ by the fuzzy satisfactionmaximizing method [29], and it can be expressed as

$$
\mu_{D}(x)=\max \left\{1-\mu_{i}(x)\right\},
$$

where $\mu_{i}(x)$ is membership of $i$ th objective function; $\mu_{D}(x)$ is objective function of fuzzy multiobjective OPF; $i \in$ $\{1,2, \ldots, m\}, m$ is the number of objective functions.

The model of fuzzy multiobjective OPF converted by (10) is described as

$$
\begin{array}{ll}
\min & \mu_{D}(x) \\
\text { s.t. } & g(x, u)=0, \quad h(x, u) \leq 0 .
\end{array}
$$

The optimal scheme of fuzzy multiobjective OPF described by (11) is the optimal solution of multiobjective $\mathrm{OPF}$, and it can make each objective function achieve satisfactory values at the same time.

\section{Modified Artificial Bee Colony Algorithm}

4.1. Artificial Bee Colony Algorithm. Artificial bee colony algorithm is a swarm intelligence optimization algorithm which simulates the behavior of honeybee swarms foraging for maximum nectar amount [18]. The honeybee swarms, food sources, and nectar amount are important parts of the $\mathrm{ABC}$ algorithm. The food sources foraged by honeybees represent a feasible solution of the optimization problem, and the nectar amount of food sources corresponds to the fitness value of associated feasible solution. The honeybee swarms include employed bees and unemployed bees, and the unemployed bees are divided into two groups: onlooker bees and scout bees. The number of employed bees $N_{e}$ is equal to the number of onlooker bees $N_{o}$ and is equal to half of the number of honeybee swarms $N_{s}$ [19]. The whole optimization process of $\mathrm{ABC}$ algorithm mainly includes initialization, employed bee phase, onlooker bee phase, and scout bee phase. And they are described by the following subsections.

4.1.1. Initialization. The $\mathrm{ABC}$ algorithm randomly generates $N_{s}$ numbers of initial swarms which are described as 
$X=\left\{x_{i j} \mid i=1,2, \ldots, N_{s} ; j=1,2, \ldots, D\right\} ; D$ is the number of parameter dimensions and is equal to the number of control variables. The initial swarms are generated as

$$
x_{i j}=x_{j L}+\operatorname{rand} \times\left(x_{j H}-x_{j L}\right),
$$

where $x_{i j}$ is the $j$ th dimension parameter of food source $i$; $x_{j H}, x_{j L}$ are the upper and lower bounds for the dimension $j$, respectively; rand is random number between 0 and 1 .

The fitness values of the initial swarms are calculated by the following expression:

$$
\text { fit }_{i}= \begin{cases}\frac{1}{1+f_{i}} & f_{i} \geq 0 \\ 1+\operatorname{abs}\left(f_{i}\right) & f_{i}<0,\end{cases}
$$

where $f_{i}$ is the objective function value of employed bee $i$, and fit $_{i}$ is the fitness value of employed bee $i$.

The higher fitness value indicates the smaller value of objective function and the better food source. The values of initial swarms are sorted from the largest to the smallest. And the corresponding food sources of the previous $N_{e}$ numbers fitness values are chosen to be considered as food sources of employed bees.

4.1.2. Employed Bee Phase. After initialization, the employed bees search for new candidate food sources $v_{i}$ within the neighborhood of the associated food sources $x_{i}$. The searching equation is described as

$$
v_{i j}=x_{i j}+R_{i j} \times\left(x_{i j}-x_{k j}\right),
$$

where $j \in\{1,2, \ldots, D\}$ and $k \in\left\{1,2, \ldots, N_{e}\right\}$ are randomly generated and $k \neq i ; v_{i j}$ is a new candidate food source; $x_{k j}$ is a randomly chosen food source different from $x_{i j} ; R_{i j}$ is random number between -1 and 1 , if a parameter value exceeds its limits, it will be fixed on its limit value.

The greedy selection mechanism is utilized to select the better solution between $v_{i}$ and $x_{i}$.

4.1.3. Onlooker Bee Phase. After all employed bees complete the searching process, the probability value that a food source will be chosen by the onlooker bee is calculated by the following expression:

$$
P_{i}=\frac{\text { fit }_{i}}{\sum_{i=1}^{N_{e}} \mathrm{fit}_{i}},
$$

where $P_{i}$ is the probability value.

Each onlooker bee chooses a food source according to the probability $P_{i}$ to search for a new food source by (14) and records the better solution by the greedy selection mechanism. By increasing the fitness value of that food source, the probability of a food source chosen by the onlooker bees increases.

4.1.4. Scout Bee Phase. After all the employed bees and onlooker bees complete their operation phase, the scout bee checks the numbers of fitness unimproved of a food source. If a food source cannot be improved further over the predetermined number of cycles limit, which indicates that the fitness value of the food source is local optima, the food source will be replaced with a new food source produced by the scout bee using (12).

4.2. Modified Artificial Bee Colony Algorithm. The ABC algorithm possesses the better global searching ability due to the employed bees and scout bee phase. The only one dimension parameter will be changed by (14) for new food sources searching, and it may find the global optimal solution in low dimension optimization problems. But the optimization efficiency will be decreased by (14) in the high dimension optimization problems, and the algorithm gets trapped in the local optimal solution easily. Therefore, the local searching ability of $\mathrm{ABC}$ algorithm is poor in the high dimension optimization problems.

The DE algorithm possesses the strong exploitation ability due to one or more dimension parameters which will be changed at the mutation operation process [30]. In order to overcome the shortage of poor exploitation capacity of $\mathrm{ABC}$ algorithm, the paper proposes the modified $A B C$ algorithm which brings the mutation and crossover operation of $\mathrm{DE}$ algorithm into the employed bee phase and onlooker bee phase instead of the searching operation of (14). The modified $\mathrm{ABC}$ algorithm is named $\mathrm{MABC}$ algorithm which combines the advantages of both $\mathrm{ABC}$ and $\mathrm{DE}$ algorithms.

On the aspect of choosing the mutation operation, the paper considers the influence of current optimal food source $x_{\text {best }}$ on searching for new food source $u_{i}$ at the $k$ th iteration. The convergence speed is improved by the effect of current optimal food source $x_{\text {best }}$. The new food source $u_{i}$ of mutation operation is generated by

$$
u_{i}=x_{\text {best }}+F \cdot\left(x_{r 1}-x_{r 2}\right) \text {, }
$$

where $u_{i}$ is a new food source generated by the mutation operation; $x_{\text {best }}$ is the current best food source having the highest fitness value; $x_{r 1}$ and $x_{r 2}$ are different food sources generated randomly, and $r 1 \neq r 2 \neq i ; \lambda$ and $F$ are scaling factor of mutation operation.

The new food source $v_{i j}$ of crossover operation is generated by

$$
v_{i j}= \begin{cases}u_{i j} & \varphi_{j} \leq \mathrm{CR} \| j=q \\ x_{i j} & \varphi_{j}>\mathrm{CR} \| j \neq q\end{cases}
$$

where $v_{i j}$ is a new food source generated by the crossover operation; $\varphi_{j}$ is random parameter of dimension $j$ in the range $[0,1]$, generated anew for each value of $j$; CR is the constant parameter of crossover operation in the range $[0,1]$; $q$ is random parameter in the range $[0, D]$ which ensures that $v_{i j}$ gets at least one parameter from $u_{i j}$.

The flowchart of MABC algorithm for solving the optimization problem is shown in Figure 2. 


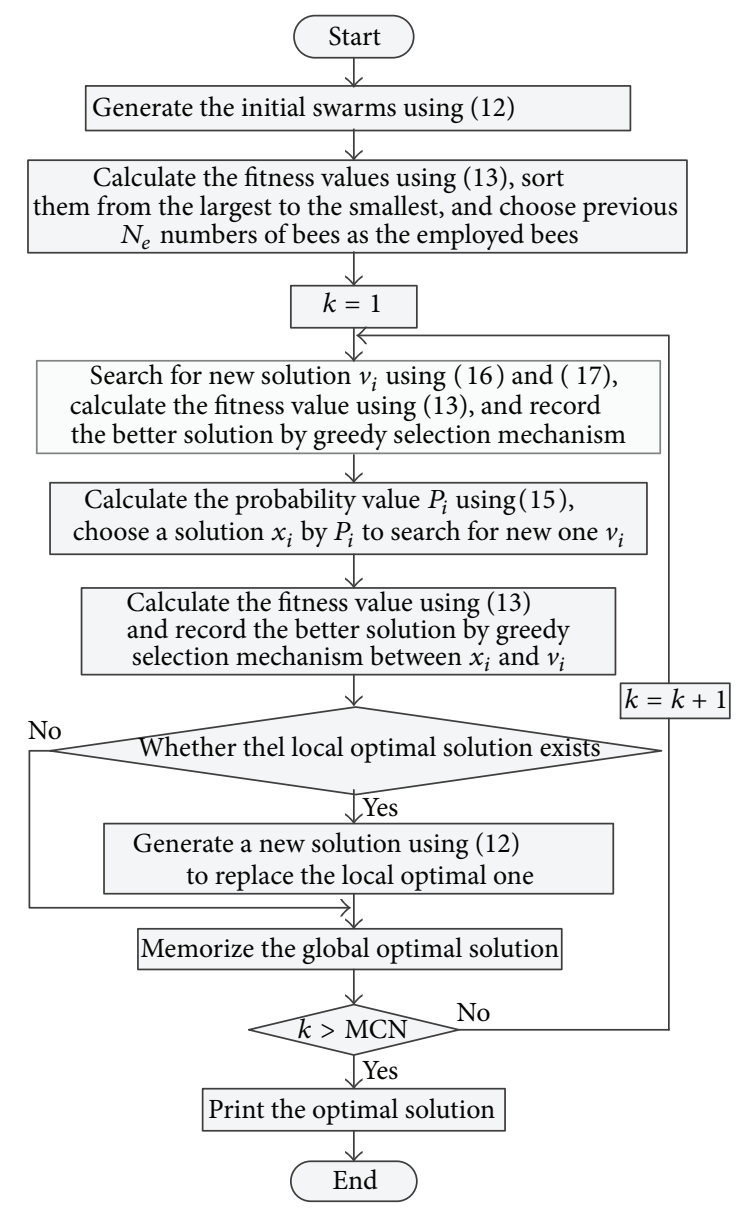

FIGURE 2: Flowchart of MABC algorithm.

\section{Fuzzy Multiobjective OPF Base on MABC Algorithm}

The proposed MABC algorithm is applied to fuzzy multiobjective OPF in power system. The capacity of optimization to multiobjective functions simultaneously can be reflected by the fuzzy fitness value which is calculated by

$$
\text { fit }_{D i}=\frac{1}{1+\mu_{D i}}, \quad i=1,2, \ldots, N_{e},
$$

where $\mu_{D i}$ is the objective function value of $i$ th food source; fit $_{D i}$ is the fuzzy fitness value of $i$ th food source; $N_{e}$ is the number of food sources.

The higher fuzzy fitness value represents the closer to optimal value of each objective function simultaneously and the better optimization capacity. The detailed calculation processes of fuzzy multiobjective OPF based on MABC algorithm are described as follows.

(1) Read the original data of power system and the control parameters of MABC algorithm.

(2) Initialization bees swarm: according to the boundary conditions, the initial bee swarms $X$ will be randomly generated by (12), $X=\left\{x_{i j} \mid i=1,2, \ldots, N_{s} ; j=\right.$ $1,2, \ldots, D\}$.
(3) Calculate fuzzy fitness value. Calculate the value of objective functions using (2) (5) based on the results of Newton-Raphson power flow calculation. Utilize (9) to convert each objective function value into corresponding fuzzy membership function values. Choose a fuzzy optimization objective function of multiobjective OPF using (10) according to each membership function value. Finally, calculate the fuzzy fitness value by (18).

(4) Select the employed bees. Sort the $N_{s}$ numbers of fuzzy fitness values obtained by the step (3) from the largest to the smallest and select food sources of the previous $N_{e}$ numbers of fitness values to be considered as employed bees.

(5) Employed bees phase: each employed bee $x_{i j}$ adopts (16) and (17) to search for new food source $v_{i j}$.

(6) Greedy selection mechanism: the fuzzy fitness value fit $\left(v_{i j}\right)$ of the new food source $v_{i j}$ is calculated by step (3). It is compared with fit $\left(x_{i j}\right)$ of corresponding food source $x_{i j}$; if the fit $\left(v_{i j}\right)$ is better than fit $\left(x_{i j}\right), x_{i j}$ will be replaced by the new food source $v_{i j}$; otherwise $x_{i j}$ is retained.

(7) Calculate probability value $P_{i}$. The probability value $P_{i}$ of each employed bee $x_{i j}$ is calculated by (15).

(8) Onlooker bees phase: a parameter is randomly generated by $n$th onlooker bee between 0 and 1 , and it is compared with $P_{i}$; if it is smaller than $P_{i}$, then the $n$th onlooker bee searches for a new food source $v_{i j}$ within the neighborhood of food source $x_{i j}$ by (16) and (17). Greedy selection mechanism is used to choose the better feasible solution between $v_{i j}$ and $x_{i j}$, and $n=$ $n+1$. Otherwise, $i=i+1$, and the $n$th onlooker bee regenerates a random parameter until it is assigned.

(9) Judge whether all the onlooker bees are assigned. If all the onlooker bees are assigned $\left(n>N_{o}\right)$, then proceed to step (10). Otherwise, return to step (8).

(10) Scout bee phase: the scout bee checks the unimproved fitness number; if a food source cannot be improved further over the number limit, the food source will be abandoned and a new food source will be generated by (12) to replace the associated food source.

(11) Record the current optimal food source, current optimal value of each objective function, and current optimal fuzzy fitness value of MABC algorithm, and the cycle parameter $k=k+1$.

(12) Cycle operation: repeat steps (5) (11) until the cycle parameter $k$ is further over maximum cycle number MCN, and then MABC algorithm is stopped and outputs the optimal solution and corresponding objective function value.

\section{Numerical Examples}

6.1. IEEE 30-Bus Test System. The IEEE 30-bus test system [14] has six generators, four transformers, and nine shunt VAR compensation devices and has a total of twenty-four 
TABLE 1: Comparisons of the results obtained by different algorithms for Case 1 .

\begin{tabular}{|c|c|c|c|c|}
\hline \multirow{2}{*}{ Method } & \multicolumn{4}{|c|}{ Fuel cost $(\$ / h)$} \\
\hline & Min & Average & Max & Standard deviation \\
\hline MABC & 800.3981 & 800.4043 & 800.4446 & 0.0105 \\
\hline $\mathrm{ABC}$ & 800.4390 & 800.5346 & 800.5844 & 0.0443 \\
\hline GSA [6] & 805.1752 & 812.1935 & 827.4950 & NA \\
\hline EGA [33] & 802.0600 & NA & 802.1400 & NA \\
\hline IEP [34] & 802.4650 & NA & NA & NA \\
\hline DE [35] & 802.2300 & NA & NA & NA \\
\hline
\end{tabular}

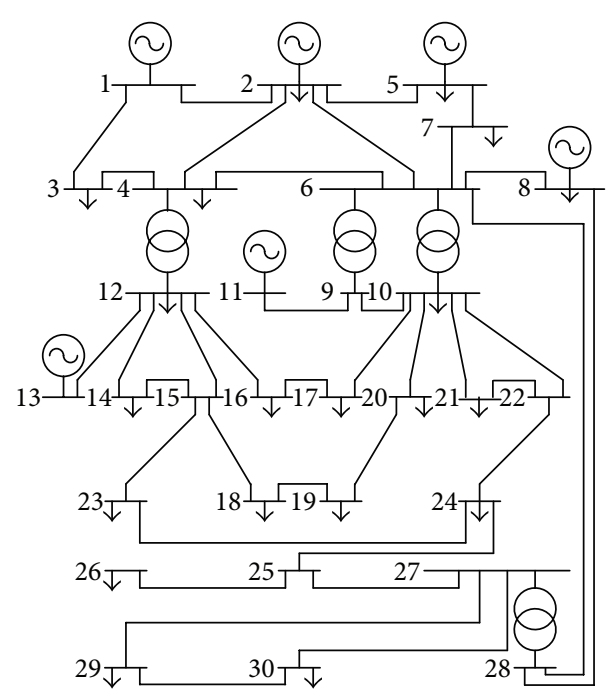

FIGURE 3: Single line diagram of IEEE 30-bus test system.

control variables. The total system demand is $283.4 \mathrm{MW}$ for active power and 126.2 MVar for reactive power. The transformer tap settings are assumed to vary in the range $[0.9,1.1]$ p.u., with step size of 0.0125 p.u.. The VAR injections of the shunt capacitors are assumed to vary in the range $[0,5]$ MVar, with step size of 1 MVar. Bus 1 is taken as slack bus, and the limits of generator buses and load buses are between 0.95-1.1 p.u. and 0.95-1.05 p.u., respectively. The Single line diagram of the system is shown in Figure 3.

6.2. Numerical Examples. The proposed MABC algorithm and the fuzzy multiobjective OPF model have been applied to the IEEE 30-bus test system. And five cases with different optimization objectives have been simulated. The different single objective OPF has been simulated in Case 1 Case 4, and the results are compared with those found by other heuristic methods. The fuzzy multiobjective OPF is stimulated in Case 5. The MABC algorithm parameters used [31,32] are $N_{s}=100, \mathrm{MCN}=300$, limit $=50, \lambda=0.6, F=0.6$, and $\mathrm{CR}=$ 0.5 .

Case 1 (minimization of total fuel cost for single objective OPF). The minimization of total fuel cost described by (2) is selected as objective function for single objective OPF. The minimum total fuel cost obtained by the proposed

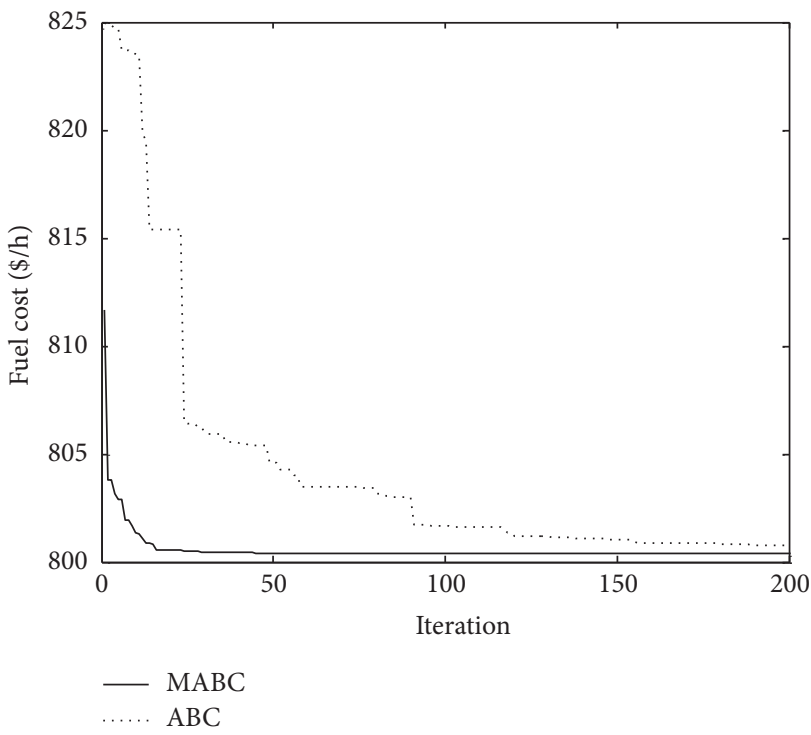

FIGURE 4: Comparisons of convergent characteristic obtained by different algorithms for Case 1 .

MABC approach over 20 independent runs was $800.3981 \$ / \mathrm{h}$ with average of $800.4043 \$ / \mathrm{h}$ and maximum of $800.4446 \$ / \mathrm{h}$, which are compared to those obtained by $\mathrm{ABC}$ and also compared to gravitational search algorithm (GSA) [6], enhanced genetic algorithm (EGA) [33], improved evolutionary programming (IEP) [34], and DE [35]. The results are given in Table 1 .

From Table 1, it can be seen that the average total fuel cost obtained by the proposed MABC approach was $800.4043 \$ / \mathrm{h}$ which is less than the $800.5346 \$ / \mathrm{h}$ obtained by $\mathrm{ABC}$ algorithm and is smaller than $812.1935 \$ / \mathrm{h}$ obtained by the GSA [6] obviously. The maximum value was $800.4446 \$ / \mathrm{h}$ obtained by MABC algorithm, and it is better than maximum values obtained by other algorithms in Table 1 . The maximum value, average value, minimum value, and standard deviation value obtained by $\mathrm{MABC}$ algorithm are better than those obtained by $\mathrm{ABC}$ algorithm. The results demonstrate that the MABC can solve the OPF problem effectively, and the optimal value obtained by MABC algorithm is better than other algorithms. The convergence characteristics of MABC and $\mathrm{ABC}$ algorithms for Case 1 are shown in Figure 4. 
TABLE 2: Comparisons of the results obtained by different algorithms for Case 2 .

\begin{tabular}{lc}
\hline Method & Emission (ton/h) \\
\hline MABC & $\mathbf{0 . 1 9 4 3}$ \\
ABC & 0.1943 \\
PSO [36] & 0.2096 \\
GA [36] & 0.2117 \\
SFLA [36] & 0.2063 \\
MSFLA [36] & 0.2056 \\
\hline
\end{tabular}

In order to compare the results by MABC with those by $\mathrm{ABC}$ obviously, Figure 4 has only described the data of previous 200 iterations. From Figure 4 and simulation data, it can be seen that when the iteration number reaches up to 20 , the fuel cost values obtained by MABC and $A B C$ are $800.5810 \$ / \mathrm{h}$ and $815.4091 \$ / \mathrm{h}$, respectively. The results demonstrate that the convergent rate of the MABC algorithm is better than that of $\mathrm{ABC}$ algorithm.

Case 2 (minimization of total emission for single objective OPF). The minimization of total emission described by (3) is selected as objective function for single objective OPF. The minimum total emission obtained by MABC is $0.1943 \mathrm{ton} / \mathrm{h}$, which are compared to those by $\mathrm{ABC}$ and also compared to those obtained by particle swarm optimization (PSO) [36], genetic algorithm (GA) [36], shuffled frog leaping algorithm (SFLA) [36], and modified SFLA (MSFLA) [36]. The results are given in Table 2.

From Table 2, it can be seen that the optimal value obtained by MABC is 0.1943 ton/h which reduces by $8.22 \%$ compared with 0.2117 ton/h obtained by GA [36] and is smaller than the values obtained by other algorithms obviously. The results demonstrate that the optimal value obtained by MABC algorithm is better than other algorithms. The convergence characteristics of the $\mathrm{MABC}$ and $\mathrm{ABC}$ for Case 2 are shown in Figure 5.

From Figure 5 and simulation data, it can be seen that when the iteration number reaches up to 20 , the minimum value obtained by MABC is already equal to global optimal value $0.1943 \mathrm{ton} / \mathrm{h}$, and at the same time the objective function value obtained by $A B C$ algorithm got tripped in local optimal value, and the $\mathrm{ABC}$ algorithm converges to global optimal value in about 160th iteration. The results demonstrate that the convergent rate of the MABC algorithm is better than that of $\mathrm{ABC}$ obviously.

Case 3 (minimization of active power loss for single objective OPF). The minimization of active power loss described by (4) is selected as objective function for single objective OPF. The minimum active power loss obtained by the MABC is $3.0819 \mathrm{MW}$, which is compared to the $\mathrm{ABC}$ and also compared to those obtained by enhanced genetic algorithm (EGA) [37], PSO [37], and DE [38]. The results are given in Table 3.

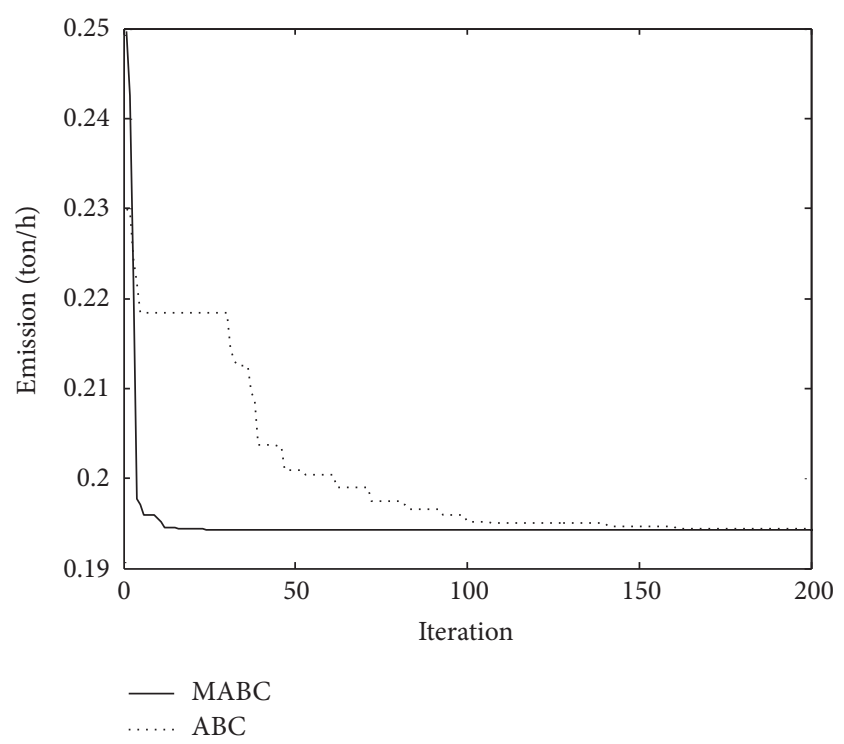

FIgURE 5: Comparisons of convergent characteristic obtained by different algorithms for Case 2.

TABLE 3: Comparisons of the results obtained by different algorithms for Case 3.

\begin{tabular}{lc}
\hline Method & Active power loss (MW) \\
\hline MABC & $\mathbf{3 . 0 8 1 9}$ \\
ABC & 3.0938 \\
EGA [37] & 3.2008 \\
PSO [37] & 3.6294 \\
DE [38] & 3.2400 \\
\hline
\end{tabular}

From Table 3, it can be seen that the optimal value obtained by MABC algorithm is $3.0819 \mathrm{MW}$ which reduces by about $15.09 \%$ compared with the $3.6294 \mathrm{MW}$ obtained by PSO [37] and reduces by about $4.88 \%$ compared with the 3.2400 MW obtained by DE [38] and is smaller than the values obtained by other algorithms. The results demonstrate that the MABC leads to better results than the other algorithms. The convergence characteristics of the MABC and $\mathrm{ABC}$ algorithms for Case 3 are shown in Figure 6.

From Figure 6 and simulation data, it can be seen that the $\mathrm{MABC}$ algorithm converges to the highest quality solution among the two algorithms in less iterations.

Case 4 (minimization of voltage deviation for single objective OPF). The minimization of voltage deviation described by (5) is selected as objective function for single objective OPF. The minimum voltage deviation obtained by MABC algorithm is 0.0841 p.u., which is compared with those obtained by $\mathrm{ABC}$ algorithm and also compared to those obtained by $\mathrm{DE}$ [15] and biogeography-based optimization algorithm (BBO) [39]. The results are given in Table 4.

From Table 4, it can be seen that the optimal value obtained by MABC is 0.0841 p.u. which reduces by $19.13 \%$ compared with optimal value 0.1040 p.u. obtained by $A B C$ 


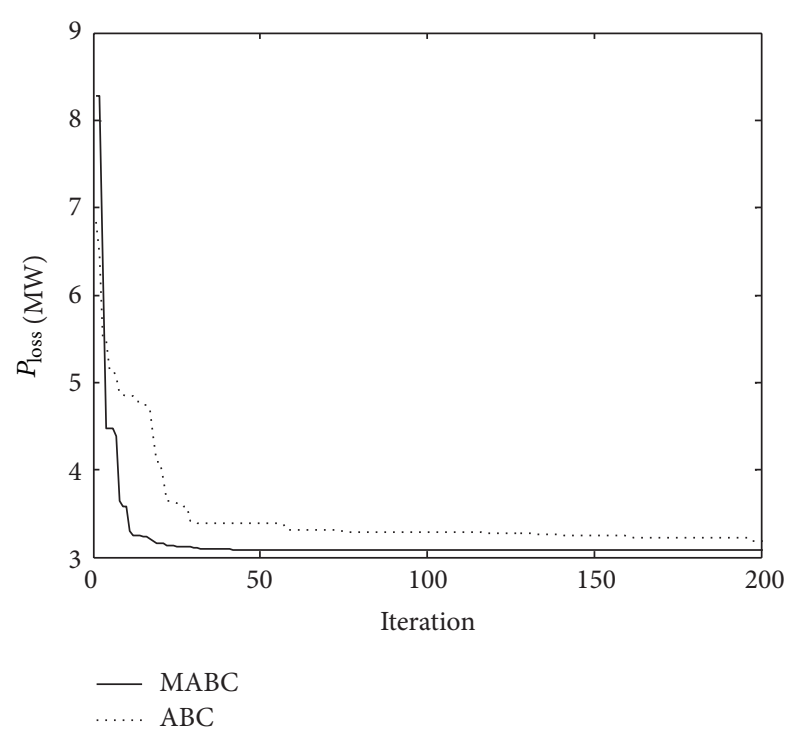

FIgURE 6: Comparisons of convergent characteristic obtained by different algorithms for Case 3.

TABLE 4: Comparisons of the results obtained by different algorithms for Case 4

\begin{tabular}{lc}
\hline Method & Voltage deviation (p.u.) \\
\hline MABC & $\mathbf{0 . 0 8 4 1}$ \\
ABC & 0.1040 \\
DE [15] & 0.1357 \\
BBO [39] & 0.1020 \\
\hline
\end{tabular}

algorithm and reduces by $38.03 \%$ compared with optimal value 0.1357 p.u. obtained by DE algorithm [15] and reduces by $17.55 \%$ compared with optimal value 0.1020 p.u. obtained by $\mathrm{BBO}$ algorithm [39]. The results indicate that the optimal value found by MABC is better than other heuristic algorithms obviously. The convergence characteristics of the MABC and ABC algorithms for Case 4 are shown in Figure 7.

From Figure 7 and simulation data, it can be seen that the convergent capacity is better than other heuristic algorithms.

From the results of single objective OPF in Case 1 Case 4 , it can be seen that the convergent capacity and the optimal value of MABC algorithm are better than ABC algorithm and other heuristic algorithms, which can demonstrate that the proposed $\mathrm{MABC}$ algorithm possesses not only the stronger exploration capacity but also the better exploitation capacity.

Case 5 (fuzzy multiobjective OPF). The optimal values obtained by Case 1 Case 4 are considered as the reference values of fuzzy membership function, and (9) and (10) are used to convert OPF with four objective functions into fuzzy multiobjective OPF which is solved by MABC algorithm. The minimum values of each objective function obtained by the proposed MABC approach and ABC algorithm over 20 independent runs for Case 5 are given in Table 5.

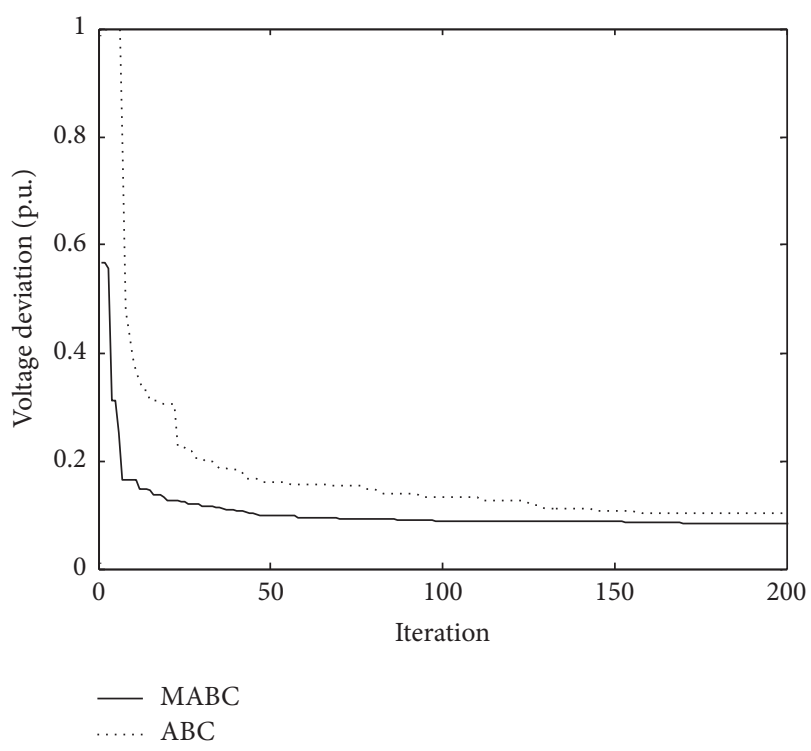

FIGURE 7: Comparisons of convergent characteristic obtained by different algorithms for Case 4 .

TABLE 5: Minimum values obtained by different algorithms over 20 independent runs.

\begin{tabular}{lcccc}
\hline \multirow{2}{*}{ Method } & \multicolumn{4}{c}{ Objective function value } \\
& FC $(\$ / \mathrm{h})$ & EM $($ ton $/ \mathrm{h})$ & $P_{\text {loss }}(\mathrm{MW})$ & $\Delta V(\mathrm{p} . \mathrm{u})$ \\
\hline MABC & $\mathbf{8 5 0 . 5 9 9 6}$ & $\mathbf{0 . 2 2 2 4}$ & $\mathbf{4 . 8 3 3 1}$ & $\mathbf{0 . 3 2 5 3}$ \\
$\mathrm{ABC}$ & 852.5226 & 0.2224 & 4.9129 & 0.3474 \\
\hline
\end{tabular}

TABLE 6: Fuzzy fitness values obtained by different algorithms over 20 independent runs.

\begin{tabular}{lcccc}
\hline \multirow{2}{*}{ Method } & \multicolumn{4}{c}{ Fuzzy fitness value } \\
& Max & Average & Min & Standard deviation \\
\hline MABC & $\mathbf{0 . 7 7 1 6}$ & $\mathbf{0 . 7 7 1 3}$ & $\mathbf{0 . 7 7 0 7}$ & $\mathbf{0 . 0 0 0 3}$ \\
ABC & 0.7645 & 0.7558 & 0.7449 & 0.0044 \\
\hline
\end{tabular}

From Table 5, it can be seen that the minimum values of each objective function obtained by MABC algorithm are $850.5996 \$ / \mathrm{h}, 0.2224 \mathrm{ton} / \mathrm{h}, 4.8331 \mathrm{MW}$, and 0.3253 p.u., respectively. And the minimum values obtained by $\mathrm{ABC}$ algorithm are $852.5226 \$ / \mathrm{h}, 0.2224 \mathrm{ton} / \mathrm{h}, 4.9129 \mathrm{MW}$, and 0.3474 p.u., respectively. The results demonstrate that the minimum values of each objective function obtained by $\mathrm{MABC}$ algorithm are better than those obtained by $\mathrm{ABC}$ algorithm for fuzzy multiobjective OPF.

The maximum value, average value, minimum value, and standard deviation value of the fuzzy fitness obtained by $\mathrm{MABC}$ and $\mathrm{ABC}$ algorithms over 20 independent runs are given in Table 6.

From Table 6, it can be seen that the maximum value obtained by $\mathrm{MABC}$ is 0.7716 which is higher than the value 0.7645 obtained by $\mathrm{ABC}$ algorithm. The minimum value and average value obtained by MABC are also higher than those obtained by ABC. The results indicate that the optimal solution obtained by MABC algorithm is better than that by 
TABLE 7: Comparison of the OPF control variables for different cases.

\begin{tabular}{|c|c|c|c|c|c|c|c|c|c|c|}
\hline \multirow{2}{*}{ Variables } & \multicolumn{2}{|c|}{ Case 1} & \multicolumn{2}{|c|}{ Case 2} & \multicolumn{2}{|c|}{ Case 3} & \multicolumn{2}{|c|}{ Case 4} & \multicolumn{2}{|c|}{ Case 5} \\
\hline & MABC & $\mathrm{ABC}$ & MABC & $\mathrm{ABC}$ & MABC & $\mathrm{ABC}$ & MABC & $\mathrm{ABC}$ & MABC & $\mathrm{ABC}$ \\
\hline$P_{G 2}$ & 48.7156 & 48.5498 & 72.5306 & 72.9516 & 80 & 80 & 79.9636 & 80 & 54.9454 & 59.6575 \\
\hline$P_{G 5}$ & 21.3816 & 21.4468 & 50 & 50 & 50 & 50 & 49.6799 & 32.1691 & 35.9487 & 37.4470 \\
\hline$P_{G 8}$ & 21.2175 & 21.9803 & 35 & 35 & 35 & 35 & 31.2334 & 25.3745 & 35 & 34.5021 \\
\hline$P_{G 11}$ & 11.9245 & 11.7107 & 30 & 30 & 30 & 30 & 30 & 23.7699 & 29.9513 & 36.8806 \\
\hline$P_{G 13}$ & 12 & 12 & 40 & 40 & 40 & 40 & 40 & 40 & 25.008 & 23.9546 \\
\hline$V_{G 1}$ & 1.0831 & 1.0840 & 1.0605 & 1.0635 & 1.0610 & 1.0619 & 1.0014 & 1.0115 & 1.0689 & 1.0719 \\
\hline$V_{G 2}$ & 1.0641 & 1.0654 & 1.0553 & 1.0541 & 1.0570 & 1.0603 & 1.0013 & 1.0071 & 1.0575 & 1.0594 \\
\hline$V_{G 5}$ & 1.0329 & 1.0339 & 1.0359 & 1.0342 & 1.0375 & 1.0392 & 1.0193 & 1.0222 & 1.0336 & 1.0306 \\
\hline$V_{\mathrm{G} 8}$ & 1.0377 & 1.0378 & 1.0424 & 1.0429 & 1.0438 & 1.0449 & 1.0033 & 0.9973 & 1.0413 & 1.0306 \\
\hline$V_{G 11}$ & 1.0269 & 1.0579 & 1.0340 & 1.0020 & 1.0159 & 1.0171 & 0.9954 & 1.0462 & 0.9930 & 0.9880 \\
\hline$V_{G 13}$ & 1.0426 & 1.0304 & 1.0584 & 1.0479 & 1.0546 & 1.0467 & 1.0142 & 0.9978 & 1.0281 & 1.0219 \\
\hline$T_{6-9}$ & 0.9625 & 1 & 0.9000 & 0.9875 & 0.9500 & 0.9625 & 0.9375 & 0.9500 & 0.9750 & 1.0125 \\
\hline$T_{6-10}$ & 0.9000 & 0.9000 & 1 & 0.9000 & 0.9250 & 0.9125 & 0.9000 & 0.9250 & 0.9625 & 0.9000 \\
\hline$T_{4-12}$ & 0.9375 & 0.9250 & 0.9750 & 0.9625 & 0.9625 & 0.9500 & 0.9625 & 0.9375 & 0.9875 & 0.9750 \\
\hline$T_{27-28}$ & 0.9125 & 0.9250 & 0.9125 & 0.9250 & 0.9125 & 0.9250 & 0.9000 & 0.9000 & 0.9375 & 0.9500 \\
\hline$Q_{10}$ & 1 & 0 & 0 & 5 & 3 & 2 & 4 & 2 & 0 & 4 \\
\hline$Q_{12}$ & 4 & 5 & 5 & 3 & 2 & 3 & 0 & 5 & 0 & 1 \\
\hline$Q_{15}$ & 4 & 5 & 5 & 5 & 5 & 4 & 5 & 3 & 4 & 5 \\
\hline$Q_{17}$ & 5 & 5 & 5 & 5 & 5 & 4 & 0 & 0 & 5 & 5 \\
\hline$Q_{20}$ & 4 & 4 & 4 & 5 & 4 & 5 & 5 & 5 & 5 & 4 \\
\hline$Q_{21}$ & 5 & 5 & 5 & 5 & 5 & 5 & 1 & 0 & 5 & 5 \\
\hline$Q_{23}$ & 2 & 2 & 1 & 2 & 2 & 3 & 4 & 4 & 1 & 3 \\
\hline$Q_{24}$ & 4 & 4 & 5 & 5 & 4 & 3 & 5 & 5 & 3 & 3 \\
\hline$Q_{29}$ & 2 & 4 & 2 & 3 & 2 & 3 & 2 & 2 & 2 & 4 \\
\hline $\mathrm{FC}(\$ / \mathrm{h})$ & 800.3981 & 800.4390 & 952.8354 & 953.6603 & 967.6192 & 967.6468 & 962.9924 & 886.8907 & 850.5996 & 852.5226 \\
\hline $\mathrm{EM}($ ton $/ \mathrm{h})$ & 0.3291 & 0.3281 & 0.1943 & 0.1943 & 0.1950 & 0.1950 & 0.1962 & 0.2131 & 0.2224 & 0.2224 \\
\hline$P_{\text {loss }}(\mathrm{MW})$ & 9.0001 & 8.9640 & 3.1611 & 3.1792 & 3.0819 & 3.0938 & 4.0211 & 5.8126 & 4.8331 & 4.9129 \\
\hline$\Delta V$ (p.u) & 0.8985 & 0.8805 & 0.9203 & 0.7925 & 0.9105 & 0.8660 & 0.0841 & 0.1040 & 0.3253 & 0.3474 \\
\hline
\end{tabular}

$\mathrm{ABC}$ algorithm. In other words, the control variables setting values obtained by MABC are able to make the system more efficient and reliable than those by $\mathrm{ABC}$ algorithm.

From Table 6, it also can be seen that the standard deviation value by MABC is 0.0003 which is smaller than the value 0.0044 by $\mathrm{ABC}$ obviously. The results demonstrate that the whole optimization capacity of MABC is obviously better than that of $\mathrm{ABC}$ algorithm.

The convergence characteristics of MABC and ABC algorithms for Case 5 are shown in Figure 8.

From Figure 8, it can be seen that the MABC algorithm converges to the highest fuzzy fitness value among the two algorithms in less iterations. In addition, the convergent characteristic curve of MABC algorithm is smoother, and the $\mathrm{ABC}$ algorithm is easily to get trapped in local optima to solve the multiobjective OPF. The results illustrate that the convergence capacity of proposed MABC algorithm is better than $\mathrm{ABC}$ algorithm.

In Case 1 Case 5, the optimal control variables and the corresponding objective function values obtained by the proposed $\mathrm{MABC}$ and standard $\mathrm{ABC}$ algorithms are given in Table 7.
From Table 7, it can be seen that the optimal solutions obtained by the proposed MABC algorithm can make the results better than those obtained by the $\mathrm{ABC}$ algorithm in different optimization objectives.

\section{Conclusions}

The paper proposes the MABC algorithm which adopts the mutation and crossover operation of $\mathrm{DE}$ algorithm instead of the searching operation of $\mathrm{ABC}$ algorithm. And the MABC algorithm is used to solve the multiobjective OPF problem which considers the total fuel cost of generating units, the emission of atmospheric pollutants, the active power losses, and the voltage deviations as the optimization objective. The simulation results show that the convergent capacity and the optimal value of $\mathrm{MABC}$ algorithm are better than $\mathrm{ABC}$ algorithm and other heuristic algorithms, which demonstrate that the proposed MABC algorithm possesses not only the stronger exploration capacity but also the better exploitation capacity and indicate the efficiency and superiority of MABC algorithm for solving the nonlinear optimization problem. 


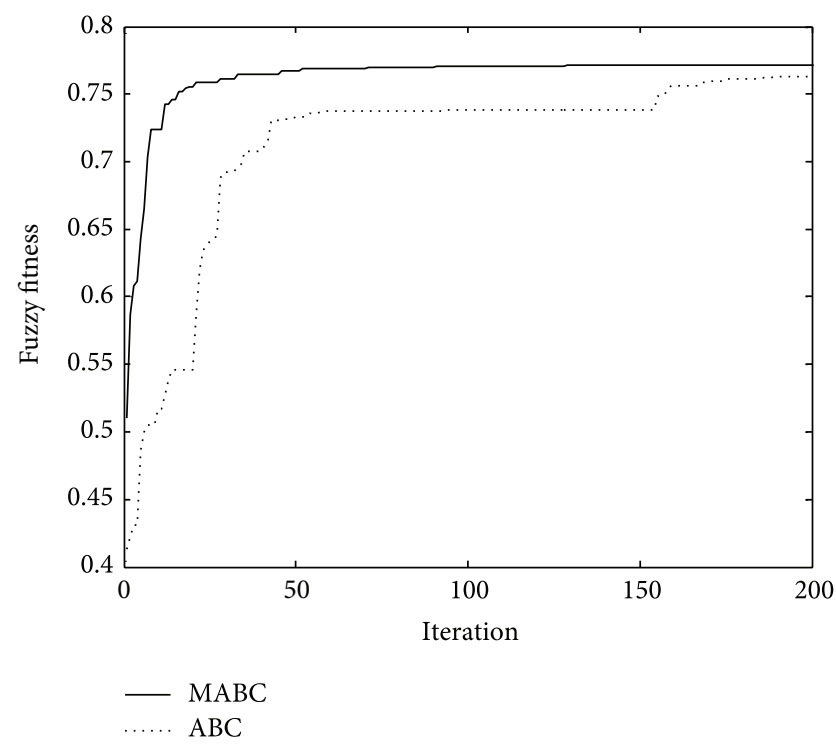

Figure 8: Comparisons of convergent characteristic obtained by different algorithms for Case 5 .

However, the influences of algorithm parameters settings on the optimization performance of MABC algorithm will be further discussed in the future researches.

\section{Conflict of Interests}

The authors declare that there is no conflict of interests regarding the publication of this paper.

\section{References}

[1] J. Carpentier, "Contribution to the economic dispatch problem," Bulletin Society Francaise Electriciens, vol. 3, no. 8, pp. 431-447, 1962.

[2] J. Carpentier, "Optimal power flows," International Journal of Electrical Power \& Energy Systems, vol. 1, no. 1, pp. 3-15, 1979.

[3] S. R. Paranjothi and K. Anburaja, "Optimal power flow using refined genetic algorithm," Electric Power Components and Systems, vol. 30, no. 10, pp. 1055-1063, 2002.

[4] J. Hazra and A. K. Sinha, "A multi-objective optimal power flow using particle swarm optimization," European Transactions on Electrical Power, vol. 21, no. 1, pp. 1028-1045, 2011.

[5] J. Pouyan, S. Arash, and R. William, "Voltage stability constrained optimal power flow," Automation of Electric Power Systems, vol. 29, no. 16, pp. 48-55, 2005.

[6] M. A. Rezaei and A. Karami, "Artificial bee colony algorithm for solving multi-objective optimal power flow problem," International Journal of Electrical Power \& Energy Systems, vol. 53, no. 1, pp. 219-230, 2013.

[7] Z. Wen and L. Yutian, "Multi-objective reactive power and voltage control based on fuzzy optimization strategy and fuzzy adaptive particle swarm," International Journal of Electrical Power \& Energy Systems, vol. 30, no. 9, pp. 525-532, 2008.

[8] O. Alsac, J. Bright, M. Prais, and B. Stott, "Further developments in LP-based optimal power flow," IEEE Transactions on Power Systems, vol. 5, no. 3, pp. 697-711, 1990.
[9] A. M. James, M. E. El-Hawary, and R. Adapa, "A review of selected optimal power flow literature to 1993.I. Nonlinear and quadratic programming approaches," IEEE Transactions on Power Systems, vol. 14, no. 1, pp. 96-104, 1999.

[10] H. W. Dommel and W. F. Tinney, "Optimal power flow solutions," IEEE Transactions on Power Apparatus and Systems, vol. 87, no. 10, pp. 1866-1876, 1968.

[11] Y.-P. Zhang, X.-J. Tong, W.-U. Felix et al., "Study on semismooth newton optimal power flow algorithm based on nonlinear complementarity problem function," Proceedings of the Chinese Society for Electrical Engineering, vol. 24, no. 9, pp. 130135, 2004.

[12] Z.-C. Qin, X.-F. Le, L. Lan et al., "Improved nonlinear predictorcorrector interior point method for optimal power flow," Automation of Electric Power Systems, vol. 29, no. 9, pp. 25-30, 2005.

[13] M. Todorovski and D. Rajicic, "A power flow method suitable for solving OPF problems using genetic algorithms," in Proceedings of the Computer as a Tool. The IEEE Region 8 (EUROCON '03), vol. 2, pp. 215-219, IEEE, 2003.

[14] M. A. Abido, "Optimal power flow using particle swarm optimization," International Journal of Electrical Power \& Energy Systems, vol. 24, no. 7, pp. 563-571, 2002.

[15] A. A. Abou El Ela, M. A. Abido, and S. R. Spea, "Optimal power flow using differential evolution algorithm," Electric Power Systems Research, vol. 80, no. 7, pp. 878-885, 2010.

[16] Y. Sun and W. Wei, "Solution of optimal power flow problem based on artificial immune algorithm," Automation of Electric Power Systems, vol. 26, no. 12, pp. 30-34, 2002.

[17] K. Ayan and U. Kılıç, "Artificial bee colony algorithm solution for optimal reactive power flow," Applied Soft Computing Journal, vol. 12, no. 5, pp. 1477-1482, 2012.

[18] D. Karaboga and B. Akay, "A comparative study of artificial bee colony algorithm," Applied Mathematics and Computation, vol. 214, no. 1, pp. 108-132, 2009.

[19] D. Karaboga, "An idea based on honey bee swarm for numerical optimization,” Tech. Rep. TR06, Erciyes University Press, Erciyes, Turkey, 2005.

[20] R. S. Rao, S. V. L. Narasimham, and M. Ramalingaraju, "Optimization of distribution network configuration for loss reduction using artificial bee colony algorithm," International Journal of Electrical Power and Energy Systems Engineering, vol. 1, no. 2, pp. 116-122, 2008.

[21] S. Ozyon, C. Yasar, B. Durmus et al., "The application of artificial bee colony algorithm for the economic power dispatch with prohibited operating zone," in Proceedings of the International Symposium on Innovations in Intelligent Systems and Applications (INISTA '12), pp. 1-5, IEEE, 2012.

[22] G. Zhu and S. Kwong, "Gbest-guided artificial bee colony algorithm for numerical function optimization," Applied Mathematics and Computation, vol. 217, no. 7, pp. 3166-3173, 2010.

[23] M. S. Alam and M. M. Islam, "Artificial bee colony algorithm with self-adaptive mutation: a novel approach for numeric optimization," in Proceedings of the IEEE Region 10 Conference (TENCON '11), pp. 49-53, IEEE, 2011.

[24] W. Gao and S. Liu, "Improved artificial bee colony algorithm for global optimization," Information Processing Letters, vol. 111, no. 17, pp. 871-882, 2011.

[25] A. Bahriye and K. Dervis, "A modified artificial bee colony algorithm for real-parameter optimization," Information Sciences, vol. 192, pp. 120-142, 2012. 
[26] W. F. Gao and S. Y. Liu, "A modified artificial bee colony algorithm," Computers \& Operations Research, vol. 39, no. 3, pp. 687-697, 2012.

[27] G. Wei-feng, L. San-yang, and H. Ling-ling, "A novel artificial bee colony algorithm based on modified search equation and orthogonal learning," IEEE Transactions on Cybernetics, vol. 43, no. 3, pp. 1011-1024, 2013.

[28] M. Liu, M. Xie, and W. Zhao, OPF of Power Network, Science Press, Beijing, China, 2010.

[29] R. Ma, "A novel bi-objective fuzzy optimal model of shortterm trade planning considering environmental protection and economic profit in deregulated power system," Proceedings of the Chinese Society for Electrical Engineering, vol. 22, no. 4, pp. 104-108, 2002.

[30] B. Liu, L. Wang, and Y.-H. Jin, "Advances in differential evolution," Control and Decision, vol. 22, no. 7, pp. 721-729, 2007.

[31] R. Gämperle, S. D. Müller, and P. Koumoutsakos, "A parameter study for differential evolution," Advances in Intelligent Systems, Fuzzy Systems, Evolutionary Computation, vol. 10, pp. 293-298, 2002.

[32] D. Haibin, Z. Xiangyin, and X. Chunfang, Bio-Inspired Computing, Science Press, Beijing, China, 2011.

[33] A. G. Anastasios, P. N. Biskas, C. E. Zoumas, and V. Petridis, "Optimal power flow by enhanced genetic algorithm," IEEE Transactions on Power Systems, vol. 17, no. 2, pp. 229-236, 2002.

[34] W. Ongsakul and T. Tantimaporn, "Optimal power flow by improved evolutionary programming," Electric Power Components and Systems, vol. 34, no. 1, pp. 79-95, 2006.

[35] K. Vaisakh and L. R. Srinivas, "Differential evolution based OPF with conventional and Non-conventional cost characteristics," in Proceedings of the Joint International Conference on Power System Technology and IEEE Power India Conference (POWERCON '08), IEEE, 2008.

[36] T. Niknam, M. R. Narimani, M. Jabbari, and A. R. Malekpour, "A modified shuffle frog leaping algorithm for multi-objective optimal power flow," Energy, vol. 36, no. 11, pp. 6420-6432, 2011.

[37] M. S. Kumari and S. Maheswarapu, "Enhanced genetic algorithm based computation technique for multi-objective optimal power flow solution," International Journal of Electrical Power \& Energy Systems, vol. 32, no. 6, pp. 736-742, 2010.

[38] M. A. Abido and N. A. Al-Ali, "Multi-objective differential evolution for optimal power flow," in Proceedings of the 2nd International Conference on Power Engineering, Energy and Electrical Drives (POWERENG '09), pp. 101-106, 2009.

[39] A. Bhattacharya and P. K. Chattopadhyay, "Application of biogeography-based optimisation to solve different optimal power flow problems," IET Generation, Transmission \& Distribution, vol. 5, no. 1, pp. 70-80, 2011. 


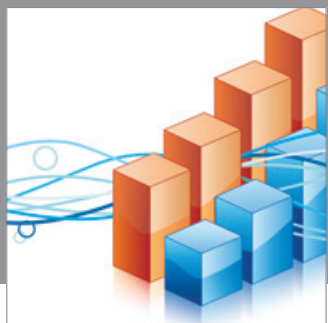

Advances in

Operations Research

mansans

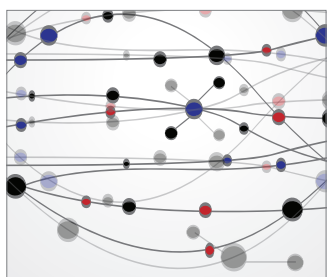

The Scientific World Journal
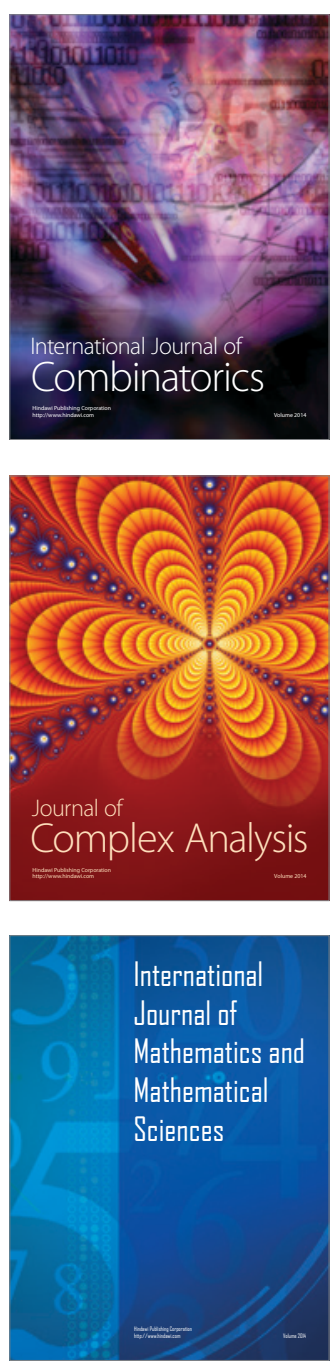
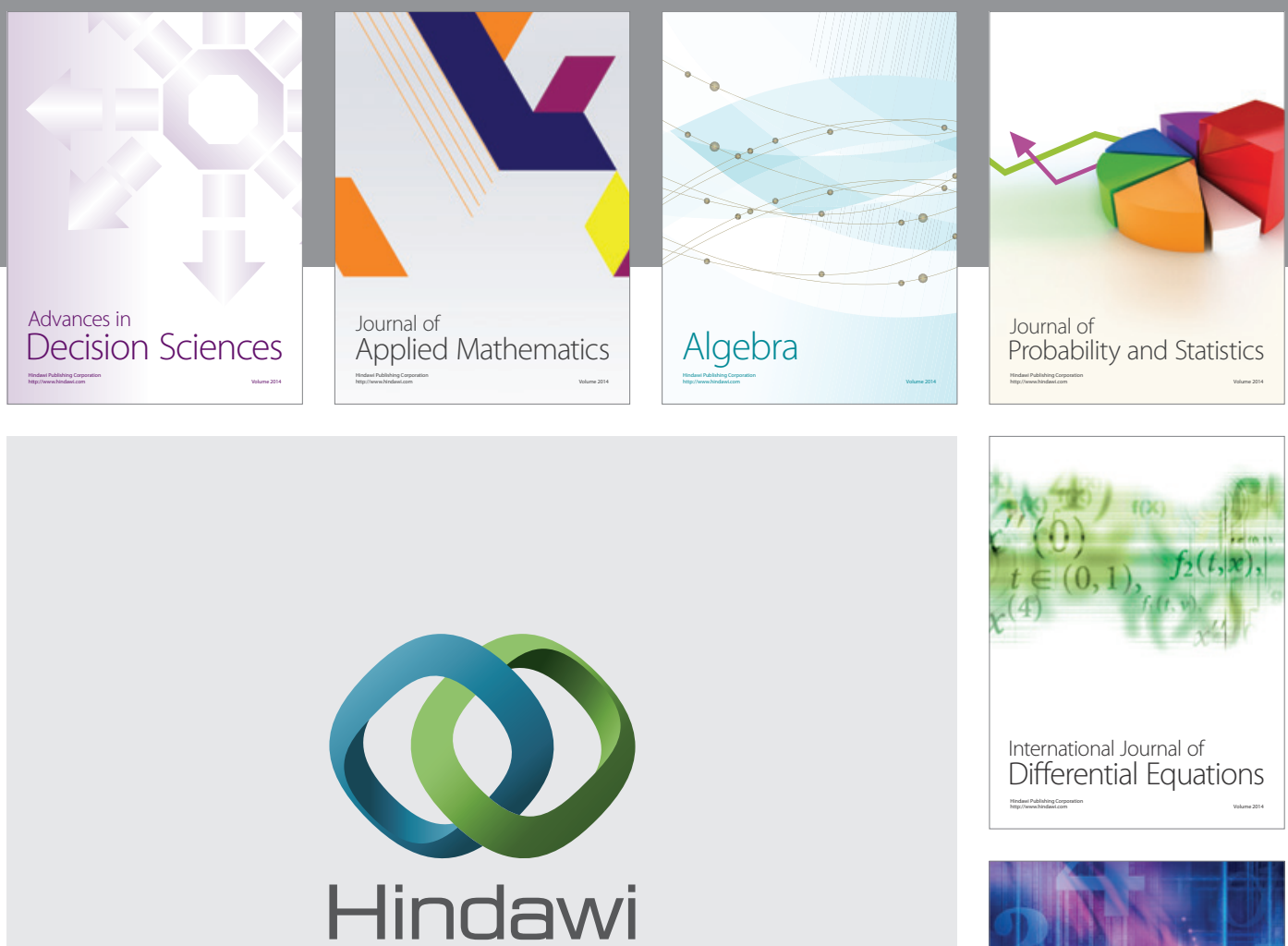

Submit your manuscripts at http://www.hindawi.com
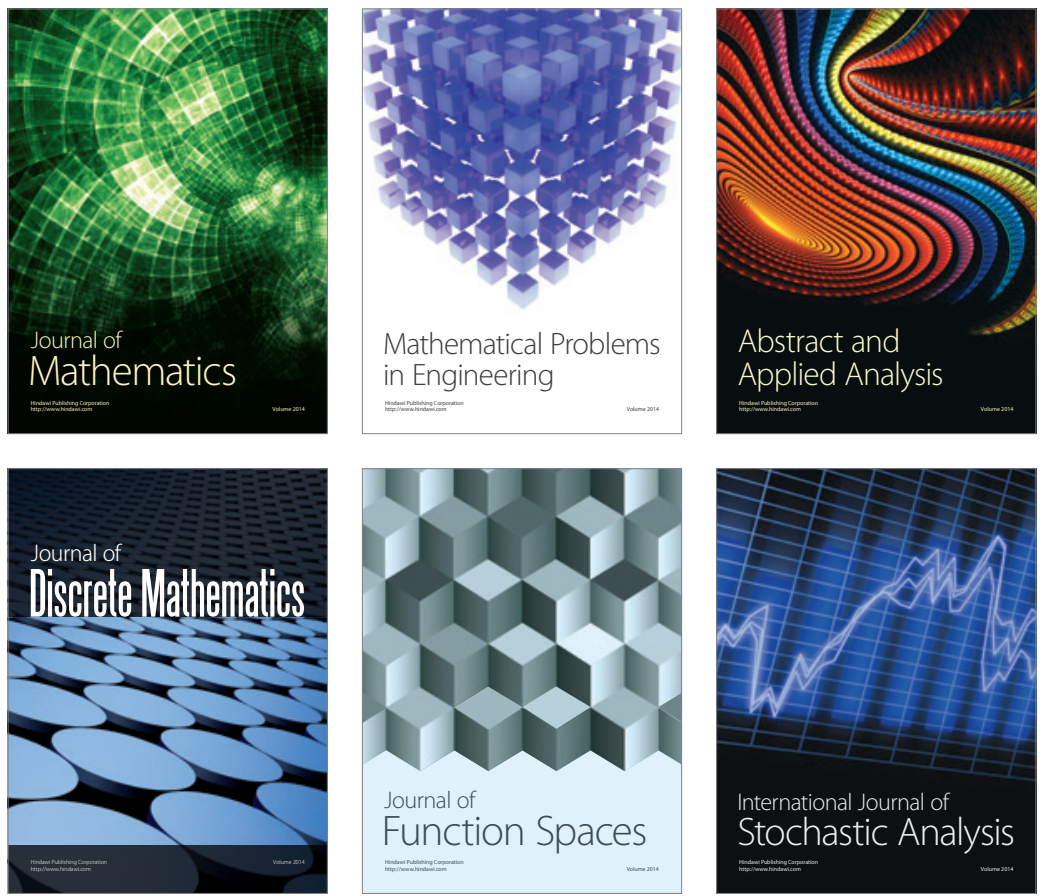

Journal of

Function Spaces

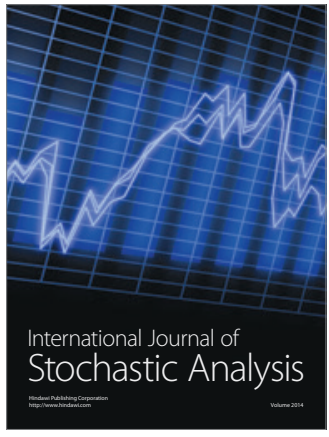

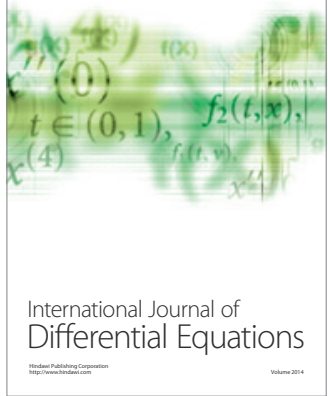
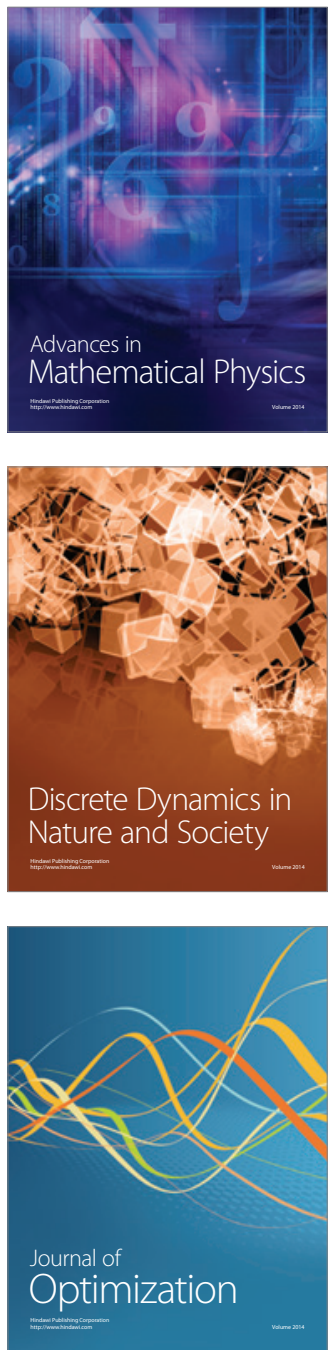